\title{
METODOLOGÍAS Y DIDÁCTICAS EN EL DEPARTAMENTO DE CIENCIAS BÁSICAS DE LA UNIVERSIDAD DE LA SALLE EN TIEMPOS DE PANDEMIA
}

\author{
Margarita Rosa Rendón Fernández* \\ Angela Cristina Zapata Lesmes** \\ Universidad de La Salle \\ https://doi.org/10.14718/9789585133846.2021.4
}

\section{Introducción}

El Departamento de Ciencias Básicas de la Universidad de La Salle es una unidad académica comprometida con la formación en ciencias exactas, físicas y naturales de estudiantes de programas propios y de otras unidades académicas de la Institución, y las áreas de las ciencias que lo constituyen son: Matemáticas, Física, Química, Biología y Estadística.

El Departamento está comprometido con el desarrollo de habilidades cognitivas, prácticas y procedimentales en los estudiantes para la comprensión de su entorno y la generación de autonomía académica, en aras del futuro desenvolvimiento en su profesión. Además, promueve el acceso y la generación de conocimiento para que profesores y estudiantes aporten alternativas de respuesta a los problemas de su línea profesional mediante ejercicios

* M. Sc. en Docencia de Química de la Universidad Pedagógica Nacional de Colombia, Licenciada en Química de la Universidad Distrital Francisco José de Caldas. Profesora investigadora de la Universidad de La Salle. https://orcid.org/0000-0002-0622-8706

* Doctora en Ciencias Biológicas y Magíster en Ciencias-Microbiología. Actualmente, decana de la Escuela de Ciencias Básicas y Aplicadas y Directora del Departamento de Ciencias Básicas de la Universidad de La Salle. https://orcid.org/0000-0002-2507-0932 
de praxis investigativa de carácter disciplinar e interdisciplinar, dentro del marco del pensamiento científico (Universidad de la Salle, 2019).

En concordancia con el Proyecto Educativo Universitario Lasallista (PEUL), la formación académica e investigativa del Departamento de Ciencias Básicas aporta en la fundamentación para

la formación de profesionales que, por su conocimiento, valores, capacidad de trabajo colegiado, visibilidad social y sentido de pertenencia, contribuyen con la búsqueda de la equidad, la defensa de la vida y del desarrollo humano integral y sustentable. (Universidad de La Salle, 2007, p. 9)

En este capítulo se presentan, de manera resumida, algunos apartes de la historia del Departamento de Ciencias Básicas y sus apuestas metodológicas y didácticas desde las áreas de conocimiento de física, química y matemáticas en medio de la pandemia con las respuestas de 20 docentes a un instrumento diseñado con tres elementos principales: (a) el propósito de la metodología, (b) los encuentros virtuales en perspectiva de las estrategias didácticas, y (c) las recomendaciones generales desde lo vivido en el desarrollo de cada espacio académico.

La experiencia de los docentes en este periodo de la vida de la humanidad, enmarcado en las necesidades de control y superación de la pandemia, aísla al docente de su estudiante en espacio, pero lo acerca a través de una pantalla de computador. Debido a esto, la apropiación del e-learning como medida de contención para la continuidad de la educación se suma a los retos de la brecha digital ya existente — y ahora más evidente-, así como a la implementación de prácticas que motiven pedagógicamente y contribuyan a disminuir la deserción por medio de cambios en las metodologías que integran el uso de herramientas tecnológicas, y con la autogestión, propendiendo por procesos de formación integral (Molinero \& Chávez, 2019).

Ahora bien, la labor pedagógica del Departamento de Ciencias Básicas en la Universidad de La Salle está altamente comprometida con la búsqueda continua de alternativas didácticas que propendan por la apropiación del conocimiento generado desde procesos de investigación teórica y práctica como fundamento conceptual para la formación profesional. En ese sentido, se asume como fundamental comunicar la ciencia desde su base epistemológica e histórica para entender los contextos en los que se generaron teorías, modelos y, más aún, cómo se ha aportado al avance tecnológico en diferentes campos de aplicación humana.

En la actual coyuntura generada por la pandemia provocada por el COVID-19, se ha hecho indiscutible asumir y proponer nuevas alternativas en el contexto de la enseñanza-aprendizaje de las ciencias exactas, físicas y naturales. Nuestro derrotero se orienta, principalmente, al desarrollo del pensamiento crítico y analítico —entre otras habilidades — en los estudiantes, 
así como al entendimiento y apropiación de conceptos, de tal forma que el futuro profesional de cualquier carrera que tenga como base estas ciencias esté en la capacidad de aportar posibles respuestas a las diversas necesidades de país, ya sea desde su trabajo individual o asociado a equipos disciplinares o interdisciplinares, construyendo y reconstruyendo de manera autogestionada, e investigando con responsabilidad y ética.

Teniendo en cuenta la reflexión continua sobre los procesos académicos de la Universidad de La Salle, y en particular del Departamento de Ciencias Básicas, esta Unidad Académica ha realizado ejercicios de análisis en relación con las estrategias y metodologías a emplear en las diferentes dimensiones de los seres humanos en esta época de aprendizajes en situaciones complejas, para seguir generando una oferta académica que responda a los compromisos para la formación de los jóvenes universitarios de los diferentes programas académicos de la Institución. Estos ejercicios y sus respectivos resultados han sido apoyados, normativizados y socializados en conjunto con instancias superiores y con el respeto que corresponde hacia la idoneidad e identidad de cada unidad académica y de cada programa de pregrado o de posgrado.

Dentro de los esfuerzos y apuestas que nuestra Institución realiza en relación con el ejercicio de la docencia en la educación superior, en el año 2015 se convocó a un grupo de docentes-investigadores, quienes, liderados desde la Vicerrectoría Académica, desarrollaron un ejercicio investigativo en relación con un proyecto institucional denominado Docencia Universitaria. Para el desarrollo de dicho proyecto, desde la Dirección del Departamento de Ciencias Básicas se organizaron las estrategias didácticas empleadas por los docentes en sus espacios académicos como insumos de base para definir las didácticas de las áreas de Matemáticas, Física, Química, Biología y Estadística, con las cuales se contaba en ese momento.

Posteriormente, se convocó a todos los docentes del Departamento para participar en un diplomado de capacitación, desarrollado desde finales del 2015 y hasta parte del 2016, que fue orientado por la Dirección de currículo de nuestra Institución con el objetivo de aportar a la formación docente en los siguientes aspectos:

- Afianzamiento de niveles de análisis, sistematización y concreción de las experiencias docentes.

- Creación de células de apoyo en los procesos de escritura de dichas experiencias.

- Generación de un documento que diera cuenta de las estrategias y metodologías privilegiadas por las diferentes áreas de las ciencias naturales, físicas y exactas que hacen parte del Departamento de Ciencias Básicas de la Universidad de La Salle. 
De otra parte, y en concordancia con la misión de nuestra Institución, en el Departamento de Ciencias Básicas se educa de manera integral y se propende por generar conocimiento para contribuir a la transformación social y productiva del país; por tanto, se hizo un trabajo que surgió como resultado de los ejercicios y desarrollos colaborativos de los docentes de cada una de las áreas del Departamento y que da cuenta, hasta ese momento, de las estrategias didácticas asumidas por cada área, dentro de los compromisos de formación en ciencias básicas de los futuros profesionales lasallistas.

Ahora bien, teniendo en cuenta el paso apresurado que la pandemia ocasionada por el COVID-19 ha generado, sin respetar las metodologías pedagógicas y didácticas de docentes jóvenes y de aquellos con más años de experiencia, todos nos hemos visto abocados a la incursión necesaria y rápida en las tecnologías de la información para desarrollar nuestras clases, así como para pensar e ingeniar estrategias didácticas y pedagógicas que den cuenta de los procesos de aprendizaje y apropiación de conceptos por parte de nuestros estudiantes y, más aún, de cómo evaluamos o damos cuenta de esos aprendizajes.

Así, para este capítulo, la base de argumentación la constituyen los testimonios de 20 docentes de las diferentes áreas del Departamento de Ciencias Básicas de la Universidad de La Salle; por tanto, escribir sobre lo que colegas hacen, viven y desarrollan en diferentes escenarios pedagógicos permite realizar un acercamiento para comprender cómo comparten el conocimiento de cada una de las áreas del saber científico, cómo lo llevan a los estudiantes, cómo inducen a la reflexión sobre lo que se va a hacer, lo que se hace y lo que se puede mejorar en el proceso de enseñanza, y cómo se da cuenta de lo aprendido y apropiado por el estudiante.

Todo lo anterior, unido a los factores educativos, los retos y las tensiones, mediados por los dominios disciplinares y conceptuales, permiten que los docentes reflexionen de manera individual y colectiva, como ruta para mejorar constantemente sus didácticas. Es un compromiso de los profesores del Departamento determinar cómo se afecta positivamente a los futuros profesionales de los diversos programas a los que se acompaña, así como determinar la incidencia directa sobre los seres humanos que se están formado en un entorno educativo, con criterios ideológicos, políticos, éticos y filosóficos lasallistas.

\section{Apuestas metodológicas y didácticas}

Retomar la trayectoria profesional de profesores universitarios en nuestro Departamento de Ciencias Básicas posibilita pensar, reflexionar y modificar la práctica docente para aportar al crecimiento de otros a través de sus propias vivencias. Por esto, a continuación presentamos los principales aportes que han generado cada una de las áreas del Departamento respecto a sus apuestas metodológicas y didácticas. 


\section{Área de Física}

El área de Física aporta al estudiante la interacción simultánea, tanto teórica como experimental, para la apropiación de los principios y leyes que la rigen, mediante la implementación de las metodologías propias del método científico.

\section{Propósito de la metodología}

La resolución de problemas es el modelo didáctico que prima en el área de Física en los diferentes espacios académicos. Esta estrategia busca el desarrollo del hábito de pensar permanentemente el mundo físico, así como el desarrollo de habilidades mentales utilizando como insumo las leyes y principios que gobiernan y regulan el comportamiento de la naturaleza.

A partir de la resolución de problemas, se crea un espacio en el cual la labor del estudiante consiste en proponer respuestas a diversas problemáticas desde cada espacio académico. Así, se privilegia el acompañamiento a los estudiantes para la generación de momentos dentro de la clase, para el análisis y la discusión que surja de las teorías, leyes, principios y modelos interpretativos de la física; así, la evaluación parte del manejo claro de conceptos para aportar a la resolución del problema enmarcado en un posible contexto de la vida real.

Los retos de la educación remota asistida por tecnologías instan a utilizar herramientas tecnológicas para dar continuidad al trabajo educativo en intenciones y objetivos, con el fin de alcanzar el propósito de la metodología adoptada y en respuesta a las necesidades de formación; compromisos asumidos responsablemente.

Esta modalidad de trabajo educativo presupone que el estudiante debe ser el centro de la enseñanza y, por lo tanto, le impone el reto de ser el constructor de su propio conocimiento desde el trabajo individual y colectivo, de acuerdo con las actividades que el docente configura para su aprendizaje. En este sentido, son propios de esta didáctica la responsabilidad ética y la madurez de autonomía intelectual del estudiante para ir más allá del cumplimiento de las actividades propuestas, de manera que se complemente el ejercicio con la explicación de los fenómenos o problemas — reales o no— sobre los cuales será indagado y enfrentado por el profesor para evaluar la apropiación de conceptos y asegurar el aprendizaje.

La metodología, evidentemente, requiere disponer de una buena infraestructura tecnológica garante de procesos fluidos de comunicación entre el estudiante y el profesor; por tanto, los recursos que más se han utilizado son los simuladores, wikis, videos, objetos virtuales de aprendizaje (OVAS), plataformas y aplicativos tipo PHET, entre otros. 
Las apuestas se realizan en espacios sincrónicos enmarcados en los horarios de clase para ello dispuestos, en los cuales tanto estudiantes como profesores concurren a un mismo espacio y tiempo, asistidos por tecnologías, con el objetivo de impedir cualquier detrimento en la calidad de la enseñanza.

\section{Encuentros virtuales desde las estrategias didácticas}

A continuación se define una de las estrategias didácticas de base del área de Física. En una sesión genérica de cualquiera de las clases impartidas se dispone de un material previo que el estudiante debe leer antes de la clase. La construcción del material es el punto más álgido de la clase que enfrenta el profesor. Debe considerar que el material de estudio resalta el tema específico, sin ser exhaustivo; y el material debe contener la reconstrucción histórica y teórica de los modelos que serán abordados — por medio de videos, simulaciones o textos—, dando cuenta de lo que se pretende que el estudiante aprehenda. Además, debe incluir un conjunto de problemas resueltos, no mayor de tres, que tengan la cualidad de abarcar la mayor parte de las diferentes aplicaciones que se le pueden presentar al futuro profesional y que apunten a la competencia que se quiere generar en el estudiante. Asimismo, el material didáctico debe contener ejercicios similares con la respuesta incluida, y el trabajo del estudiante es contrastar y analizar las respuestas obtenidas con las dadas en el texto, para posteriormente discutirlas con el profesor, lo cual requiere que el material de estudio tenga una didáctica específica para cada tema.

Durante el encuentro remoto, con los estudiantes presentes, la metodología empleada inicia con la realización de una explicación sucinta del contenido del material, y enseguida se aborda el desarrollo de los ejercicios. Luego se le pregunta a los estudiantes respecto a uno de los ejercicios del material enviado para lectura previa, en donde el estudiante tiene la posibilidad de comentar acerca del procedimiento utilizado y la solución de este. Se continúa en esa misma dinámica para el abordaje de los otros ejercicios, preguntando y contrapreguntando sobre las soluciones, e insistiendo en el análisis de las respuestas. Con esta metodología de clase se procura el desarrollo de competencias en manejo conceptual situado, comprensión, análisis calidad y rapidez de respuesta como elementos de base en un proceso de aprendizaje.

Finalmente, y para dar cuenta de lo sucedido durante el espacio de clase, se solicita a los estudiantes el envío de las soluciones de los ejercicios resueltos en clase y los que no se alcanzaron a desarrollar. Se realiza una evaluación y en algunas ocasiones se les invita a realizar procesos de autoevaluación con el propósito de determinar bajo cuáles criterios se asignan su nota. Luego se evalúa el número de respuestas correctas o las fallas que corrigieron para obtener el resultado correcto y calcular su propia calificación. Para concluir el ejercicio, de 
manera conjunta, en la siguiente sesión se hace un breve repaso de lo ocurrido, y se evalúa a través de una pregunta o problema contextualizado y sobre el tema visto.

\section{Recomendaciones generales}

Sin lugar a duda, esta metodología requiere tiempo de dedicación a cada espacio académico por parte del docente para la preparación del material que se presentará en cada uno de los encuentros virtuales.

En específico, para los profesores del área de Física, así como para otros docentes, la metodología virtual no permite el diálogo directo docente-estudiante, lo que convierte esta relación en un espacio frío, al que le hace falta la interacción directa de persona a persona que permite interpretar expresiones de los seres humanos desde su interacción y, en esa perspectiva, enriquecer los procesos de enseñanza-aprendizaje. Sin embargo, se considera que, en términos de aprendizaje de elementos teóricos, no hay mucha diferencia entre una clase presencial versus una clase virtual.

De otra parte, de acuerdo con el reporte que realiza la Coordinación del área de Física, se recoge la percepción de los estudiantes respecto al trabajo de laboratorio, que en esta época de pandemia no admite la asistencia presencial; según ellos, estas clases no superan lo que ya han realizado en sus colegios, lo que demuestra el gran interés por retornar a la presencialidad. En ese sentido, se ha optado por realizar experiencias de laboratorio utilizando recursos que se encuentran en sus casas y por medio de videos para el análisis y obtención de datos que les permitan construir un modelo que dé cuenta del fenómeno analizado.

Aun así, también se pueden reportar como ventajas del trabajo virtual el hecho de que en estas clases: (a) la evaluación en plataforma permite la entrega inmediata de resultados; (b) se facilita el seguimiento al proceso de aprendizaje por parte del estudiante y del grupo de clase; (c) se hace seguimiento, en tiempo real, de las entregas de trabajos, talleres, y demás compromisos por parte de los estudiantes; y (d) se facilita brindar respuestas a las inquietudes de los estudiantes, lo cual facilita a su vez el reforzamiento de los conocimientos y conceptos estudiados.

Finalmente, dentro de lo que se podría considerar como desventajas está el hecho de que el estudiante fácilmente puede estar siendo saturado con trabajo, teniendo en cuenta los diferentes espacios académicos a los cuales debe responder. Este es uno de los elementos a tener en cuenta para no agobiar al estudiante y permitirle realizar sus procesos de aprendizaje adecuadamente. 


\section{Área de Química}

La química es una disciplina de desarrollo constante que permite la explicación de los fenómenos que nos rodean, en los que la transformación de los elementos y compuestos es fundamental para entender su contribución al bienestar de la humanidad.

\section{Propósito de la metodología}

El área de Química está comprometida con el acompañamiento a los estudiantes para la apropiación de las metodologías científicas, mediante la comunicación de información de carácter científico con tres estrategias didácticas que propenden por un aprendizaje colaborativo: el acercamiento al trabajo experimental en ciencias, la reconstrucción colectiva del conocimiento, y la aproximación a la práctica investigativa.

Estas tres estrategias favorecen el trabajo en equipo de los estudiantes y el desarrollo de habilidades tanto individuales como grupales a partir del análisis, discusión y consolidación explicativa de los diferentes fenómenos que se abordan. Su fundamento está en despertar el interés por la investigación científica y contribuir al desarrollo de algunas habilidades propias de las metodologías científicas.

Estas estrategias, ante la necesidad de garantizar calidad de los procesos educativos en los diferentes espacios académicos de química en tiempos de pandemia, han permitido integrar y adoptar diversas maneras de garantizar la comunicación con los estudiantes y continuar con el seguimiento, usando los medios tecnológicos como herramienta a nivel sincrónico y asincrónico, y por medio de diferentes plataformas que garantizan la enseñanza de la química.

Entre las herramientas especializadas que han permitido desarrollar sesiones tanto en lo teórico como en lo práctico del laboratorio se encuentran, por ejemplo, los laboratorios virtuales en páginas como Biomodel ${ }^{2}$, Vlab $^{3}$ y Labxchange ${ }^{4}$, en las cuales se elaboraron guías con información sobre la introducción al tema, objetivos, materiales, metodologías, forma de tratar datos y bibliografía. Adicionalmente, se han desarrollado algunos encuentros de clase con simuladores Phet, software interactivo, videos de Youtube y ovas como complemento de los contenidos. Igualmente, se han desarrollado presentaciones interactivas H5P para que el estudiante contraste si comprende o no lo visto en la clase.

2 http://biomodel.uah.es/lab/

3 https://vlab.amrita.edu/

4 https://www.labxchange.org/library/items/ 
De otra parte, se realizaron lecturas de artículos científicos en inglés, la resolución de cuestionarios en grupo, y el uso del software Padlet para publicar la solución de un ejercicio y visualizar cómo otros ejercicios han sido solucionados por otros estudiantes. Finalmente, cabe destacar el uso de otras herramientas, como los canales de comunicación a través de WhatsApp.

Todo lo anterior favoreció la motivación de los estudiantes y la asociación de los contenidos con problemas propios de sus profesiones, lo cual ha servido para acercarlos a la metodología científica mediante la solución de situaciones problémicas que les permitieran describir lo observado, tomar, registrar y organizar datos, plantear y ejecutar procedimientos, analizar resultados, contrastar teorías, y llegar a conclusiones.

\section{Encuentros virtuales desde las estrategias didácticas}

En los diferentes espacios de química mediados por un acompañamiento remoto se privilegió el aprendizaje colaborativo entre educandos y docentes con el fin de alcanzar el desarrollo de competencias y la profundización de los conceptos. Para esto, se retomó, revisó y modificó algunos materiales que se tenían de presentaciones en Power Point para las clases presenciales con la finalidad de ajustar el tiempo, para que fuera un poco más corta la clase. Asimismo, se disminuyó el número de diapositivas, y otras fueron reemplazadas con un video en español o inglés para que no siempre los estudiantes escucharan al mismo maestro. Además, se diseñaron otros materiales y actividades con talleres para realizar durante las sesiones virtuales.

Durante la clase se mostraba la temática y el objetivo o la importancia de la misma y su aplicación, y luego se daba la explicación correspondiente, realizando un constante intercambio de preguntas con los estudiantes. Después de la clase se preguntaba a los estudiantes sobre lo desarrollado y presentado, y si tenían dudas, y se realizaba retroalimentación general y particular sobre cada actividad. Para el desarrollo de los laboratorios se emplearon ejercicios de simulación, laboratorios controlados y trabajos en casa guiados para generar aprendizaje experiencial. Lo anterior, procurando mantener las diferentes estrategias del área, como la reconstrucción colectiva del conocimiento y aproximación a la práctica investigativa.

Con esto, el propósito fue favorecer el trabajo en equipo de los estudiantes, desarrollar habilidades individuales y grupales, contrastar lo consultado, y realizar las diferentes actividades durante el avance en las temáticas, otorgando espacios en cada encuentro para resolver dudas.

Las unidades temáticas permiten la comprensión de algunos fenómenos y avances alcanzados gracias al conocimiento de cada uno de los elementos químicos presentes en el planeta Tierra. En cada tema, el estudiante podía encontrar videos de entre cinco y diez minutos 
de duración; además, contaba con documentos descargables que le permitirían ampliar los contenidos abordados en $P D F$. Al finalizar cada tema, el estudiante debía responder los cuestionarios y evaluaciones que le permitieran fortalecer los conocimientos aprendidos. Por otra parte, para generar un ambiente de aprendizaje que facilite la adquisición del conocimiento y el desarrollo de las habilidades, se necesitó: (a) textos - por ejemplo, textos universitarios, revistas o artículos científicos-; (b) medios audiovisuales — por ejemplo, cuadros, películas, videos-, y (c) software interactivo, ovAs, y otros.

Para evaluar los aprendizajes, se propusieron diferentes actividades de autoevaluación, coevaluación y heteroevaluación, dentro de las cuales se encuentran la evaluación de presaberes — conducta de entrada propuesta como la primera actividad del curso- , foros de discusión o debate, lecciones evaluativas, tareas y juegos. Al finalizar cada unidad, se contempla la aplicación de un cuestionario evaluativo de las temáticas estudiadas. Después de cada prueba realizada en Moodle, Microsoft Forms u otros recursos, los estudiantes podían ver las respuestas que no acertaron, pero no se les daba la respuesta, con el propósito de que, posteriormente, revisaran sus apuntes, libros, o preguntaran a sus compañeros para encontrar la respuesta correcta, a partir de su propio análisis.

Al finalizar el curso, se evaluó mediante la presentación de un examen final sobre todas las temáticas abordadas. A nivel teórico, se desarrollaron ejercicios de análisis, reflexión crítica, búsqueda de información en fuentes confiables, análisis de casos y problemas, e investigación —indagación-; y a nivel práctico, se emplearon ejercicios de simulación, laboratorios controlados y trabajos de campo — en casa — guiados para generar aprendizaje experiencial - como entrevistas, diarios de campo, etc.-. Además, se incluyó la presentación del proyecto de curso, en donde durante todo el semestre generaron y presentaron avances; para esto, aplicaron el método científico con la temática escogida — que era sobre problemas en nuestro país, como el "alimento", por ejemplo—, lo cual les permitió relacionar los diferentes temas del curso y describir lo que observaban, tomar, registrar y organizar datos, plantear y ejecutar procedimientos, analizar resultados, contrastar teorías, y llegar a conclusiones para luego comunicar, de forma oral y escrita, la propuesta de solución a los compañeros, por lo general por medio de un producto final que consistía en un video de 15 minutos en el que contaban cómo desarrollaron el tema, las normas consultadas y las fuentes que les permitieron construir la propuesta.

\section{Recomendaciones generales}

Con estas metodologías virtuales, el área de Química sigue con el objetivo, en los diferentes espacios académicos, de realizar un acompañamiento a los estudiantes para que se sientan parte de un equipo, haciéndoles conocer lo que se espera que ellos aprendan, aportando en 
el desarrollo de sus habilidades, proporcionándoles fuentes para que puedan comunicar lo que aprenden —o las dificultades que tengan—, y participando responsablemente a nivel individual o también en trabajo en equipo; de ahí la importancia de la socialización tanto de la guía de trabajo como de los ejercicios de la misma. Por otra parte, la resolución de las dudas en los talleres y guías de laboratorio se vuelve indispensable, y la matriz de evaluación que se realiza en cada uno de los cortes permite corregir y mejorar los procesos y actividades desarrollados, permitiendo un mejor desempeño a medida que va transcurriendo el semestre.

De otro lado, este tipo de metodologías virtuales permite a los estudiantes tímidos participar al menos por chat o también oralmente con la cámara apagada; sin embargo, lo ideal sería que todos los estudiantes tuvieran la cámara encendida durante la clase, o al menos cuando participan o cuando se requiere. La voz de los estudiantes de necesitar el desarrollo de los laboratorios a nivel presencial para lograr una mejor comprensión es un sentir también de los docentes para que los estudiantes puedan ver las propiedades de las sustancias y sus transformaciones con todos los sentidos: la vista, el oído, el olfato, el tacto, y el gusto, además de desarrollar valores de responsabilidad, de conciencia ecológica y de respeto a las normas de seguridad, entre otras.

También es importante mencionar que pasar de grupo en grupo para supervisar es más demorado con las plataformas que cuando se hace de forma presencial, por lo tanto, las actividades grupales tardan mayor tiempo que cuando se realizan en forma presencial. Además, estas metodologías a veces hacen que los estudiantes se distraigan más fácil, por lo que hay que estar preguntando constantemente y captando la atención continuamente.

Por último, cabe decir que para implementar este tipo de metodologías se requiere un esfuerzo muy grande de creatividad para lograr la participación y compromiso por parte de los estudiantes, puesto que el acompañamiento es sin rostros, muy frío, y las respuestas o participación espontanea de parte de ellos es muy escasa.

También, se pueden encontrar problemas con la conectividad o posibles inconvenientes con la plataforma que se usa o con los documentos o software con los que se desea desarrollar el tema, pues muchos estudiantes presentan problemas técnicos y a veces no lo comunican. Finalmente, se espera colaboración entre pares de colegas, o mayor comunicación para lograr mejores materiales o compartir diversas fuentes que enriquecen los diferentes espacios académicos. 


\section{Área de Matemáticas}

El área de Matemáticas busca que los estudiantes representen, analicen y resuelvan situaciones problémicas mediante el desarrollo de un lenguaje simbólico y de un sistema conceptual lógicamente estructurado y organizado.

\section{Propósito de la metodología}

El área de Matemáticas pretende desarrollar la habilidad para que los estudiantes puedan comunicarse matemáticamente, es decir, expresar ideas, interpretar y evaluar, representar, usar consistentemente los lenguajes natural y simbólico, describir relaciones y modelar situaciones cotidianas; así mismo, se busca que los estudiantes sean capaces de manejar la manipulación —exploración de ejemplos, casos particulares-, la formulación de conjeturas —núcleo del razonamiento matemático, proponer sistemáticamente afirmaciones que parecen ser razonables, someterlas a prueba y estructurar argumentos sobre su validez-, la generalización — descubrir una ley y reflexionar sistemáticamente sobre ella—, y la argumentación - explicar el por qué estructurar argumentos para sustentar generalización, someter a prueba, explorar nuevos caminos-.

De igual forma, se espera que los estudiantes potencien habilidades desde el trabajo matemático para ponerlos en juego en el momento de resolver problemas en varios contextos. Es por ello que el área de Matemáticas privilegia para los procesos de enseñanza y aprendizaje la resolución de problemas, pues esta permite poner en juego el conocimiento matemático, su uso y las habilidades de los estudiantes.

De acuerdo con Pozo (1993), en la resolución de problemas se involucran, entre otros, factores de tipo cognoscitivo y conductuales: por una parte, el proceso cognoscitivo hace referencia al dominio de los conocimientos específicos, y, por otra, el factor conductual se relaciona con las habilidades y destrezas básicas del estudiante. Por esta razón, los problemas se abordan cuando el estudiante construye unos aprendizajes básicos respecto a conceptos y procedimientos matemáticos y de otras disciplinas, y este es un factor que implica explorar la interdisciplinariedad para poner en uso el conocimiento matemático, de tal forma que se establezcan las conexiones pertinentes con otras ciencias o con el mundo de lo cotidiano.

Desde la teoría de Polya (1965), la resolución de problemas se basa en la solución, por parte de los estudiantes, de situaciones no resueltas, y su planteamiento debe ser planeado por los profesores, en algunos casos partiendo de problemas originales, y en otros haciendo adaptaciones desde los libros de texto a partir de una metodología adecuada. Para su formulación, se debe iniciar desde el planteamiento y comprensión de los problemas, para lo cual se deben 
resolver preguntas como “¿cuál es la incógnita?”, ¿¿cuáles son los datos?”, “¿cuál es la condición?"; y, posteriormente, continuar con la concepción de un plan, resolviendo preguntas como “¿qué puedo encontrar?”, “¿cómo puede ser útil una idea?”, “¿qué puedo hacer con una idea incompleta?” o “¿qué gano haciendo esto nuevamente?”. Más adelante, se debe seguir con la ejecución del plan, con preguntas como "¿por dónde debo empezar?", "¿qué puedo hacer?”, “¿qué gano haciendo esto?”; y, por último, se debe hacer la visión retrospectiva o reflexión, con preguntas como “¿pude verificar el resultado?”, “¿pude verificar el razonamiento?", “¿pude obtener el resultado de un modo distinto?”, “¿pude verlo de golpe?” y “¿puedo utilizar este resultado o este método para resolver otros problemas?". En esta estrategia, el profesor puede decidir en algunas fases si el trabajo se hace en forma individual o grupal.

Esta estrategia permite potenciar en los estudiantes el trabajo colaborativo en la mayoría de sus fases, facilita la discusión entre los equipos de trabajo, y a través de ella se abre un campo de posibilidades para generar en los estudiantes una motivación hacia el estudio de las matemáticas desde el planteamiento de problemas retadores.

\section{Encuentros virtuales desde las estrategias didácticas}

Los docentes manifiestan que con la virtualidad solo se reemplazó el espacio presencial por lo virtual, pero no la forma de abordar el desarrollo de los espacios académicos, ya que: (a) el tema a desarrollar por semana se tenía planificado desde comienzo de semestre; (b) el material de apoyo incluye diapositivas que ya se tenían en clase presencial, con ideas similares para el desarrollo del contenido, aunque se incluye el uso de software dentro de la misma sesión si el tema lo amerita; (c) se desarrolla el contenido preparado, como en clase presencial, y se refuerzan algunos conceptos importantes mediante preguntas que los estudiantes deben ir contestando; y (d) la clase finaliza indicando la tarea de consulta y los ejercicios de refuerzo que deben realizar, e igualmente se especifica si los deben entregar o no.

Ahora bien, las intervenciones por parte de los estudiantes se emplean las herramientas de Collaborate dispuestas en Moodle, tales como el chat, el foro y el audio, y en cuanto a las asignaciones para la casa, estas se orientan en la clase y se entregan a través del correo institucional o foro de inquietudes del aula virtual.

Respecto al desarrollo de las sesiones, en el inicio se hacen preguntas sobre la temática trabajada en la sesión anterior — con el fin de que los estudiantes manifiesten sus claridades y sus inquietudes-, el docente hace la retroalimentación correspondiente, y posteriormente introduce la nueva temática —empleando situaciones cercanas a los estudiantes, mostrando dónde y cómo pueden utilizar el concepto a trabajar—, se indica el objetivo de aprendizaje 
a desarrollar, y se les pregunta a los estudiantes si conocen de situaciones similares. En el desarrollo, el docente plantea el nuevo concepto usando diferentes representaciones, en algunas ocasiones parte de la definición, otras veces desde un ejemplo, o utiliza alguna gráfica para exponer el tema; y luego de su intervención asigna un ejercicio exploratorio para verificar dónde se tienen dificultades y socializa las diversas respuestas de los estudiantes. Al final, el docente hace una retroalimentación de lo trabajado a lo largo de la sesión y permite que los estudiantes manifiesten sus inquietudes; luego cierra la sesión de la clase haciendo una asignación.

Como se puede ver, las metodologías en el área de Matemáticas deben estar encaminadas al análisis de situaciones y solución de problemas, no tanto a enfatizar tanto en procesos algorítmicos. No obstante, se debe procurar la interacción con los estudiantes y comprender que no todos los tienen el mismo potencial de acceso a las sesiones remotas, por lo tanto, la asistencia debe gestionarse en varios momentos. La grabación de las sesiones y el acceso a ellas por parte de los estudiantes es un buen recurso para retomar los temas siempre que se necesite.

Ejemplos de algunas herramientas utilizadas durante las clases remotas son el programa Socrative, que es rápido de aplicar y de fácil uso, pero muchos estudiantes, en especial los más pequeños, no toman con seriedad que es parte de un aprendizaje integral. Por otra parte, el uso de canales como Youtube es un tema complejo, pues esta plataforma ofrece muchos videos, y no siempre son de buena calidad; el docente debe colaborar en darles una guía sobre cuáles pueden ser más exactos y eficientes para el aprendizaje, pues hay un gran número de información y videos sobre el mismo tema. De hecho, se puede decir que muchos de estos videos se quedan cortos cuando hay que trabajar temas más especializados, como lo es el cálculo vectorial.

\section{Recomendaciones generales}

Respecto al uso de plataforma Moodle, se puede decir, inicialmente, que se gasta mucho tiempo preparando los temas, pero como ventajas se encuentra que sirve de comunicación comunitaria - es decir, todos se informan de las dudas que tienen otros y esto facilita la comunicación dentro del grupo-, que brinda información rápida y personalizada, y que sirve como apoyo en el manejo de información. No obstante, como desventajas encontramos que a veces, de tantos correos, se perdían comunicaciones de estudiantes o de las directivas —en una hora se podía recibir entre 20 y 30 correos-, que facilita el trabajo de la clase - lo cual hace las clases más dinámicas_-, que es posible acceder desde el celular —lo que lo hace portable, de fácil acceso y no sobrecarga el sistema-, y que no hay gasto innecesario de papel. 
Por otra parte, se encuentra que, en un principio, el uso de un tablero en la casa no fue la mejor herramienta para el desarrollo de los encuentros con los estudiantes, pero esto se solucionó con el uso de la tableta. No obstante, como desventajas, muchas veces la tableta no se actualizaba en tiempo real, lo que causaba que reiterativamente debía refrescar la página o esperar a que se actualizara — pero eso ya es cuestión de paciencia—; además, no todos los estudiantes la usaban, aunque se les insistía que podían acceder a ellas, que solamente debían decir cuándo les quedaba mejor; no obstante, la versión gratuita deja marcas de agua, lo que genera distracción, y no se sabe si el estudiante está o no poniendo cuidado a clase en tiempo real.

Uno de los problemas más importantes es que, si los estudiantes ven el video posteriormente y les surge una duda, no van a tener espacio para realizar preguntas directamente. Una alternativa que surge para solucionar este punto es que se cree un espacio — con horas o tiempo asignado a la carga académica - para resolver dudas o preguntas exclusivamente a los estudiantes con estas características.

Por otra parte, un aspecto para resaltar es que los estudiantes tienen acceso a los solucionarios de los libros, y muchos de ellos se dedican a transcribir literalmente — con errores y todo- lo que aparece en esos sitios web gratuitos. Así que la manera para solucionar esto es extraer ejercicios de libros que sean muy viejos, o, de ser posible, inventarse unos propios.

Adicionalmente, la copia que hacen en los parciales es evitable solo si el profesor está dispuesto a calificar por mucho tiempo, o si ya tiene a su disposición un gran banco de preguntas. Se puede dejar dos preguntas abiertas para que los estudiantes, con su puño y letra, tengan que escribir la solución. Esto disminuye un poco la cantidad de plagio que hacen en dichos exámenes.

Por último, cabe mencionar que es confuso tanto para el estudiante como para el profesor el tema de la "flexibilidad", pues resulta confuso tener amarrado un reglamento estudiantil que se hizo pensando en presencialidad y no en virtualidad, y es difícil adecuar, sobre la marcha, un plan de trabajo cuando se trae consigo la calificación de productos y no la evaluación de un proceso.

\section{A modo de conclusión}

En este capítulo se compartieron acercamientos a la didáctica aplicada durante la época de aislamiento forzado provocado por la pandemia por el COVID-19. Las didácticas fueron desarrolladas de forma remota desde referentes históricos y culturales que marcaron la vida del docente y sus prácticas en tres de las cinco áreas de las ciencias que se comparten en el 
Departamento de Ciencias Básicas de la Universidad de La Salle, a saber: Física, Química y Matemáticas.

En esta etapa de retos para la formación académica de estudiantes, los docentes hicieron uso de su conocimiento y creatividad para promover distintos tipos de aprendizaje acordes con su experiencia profesional, su lógica e ideas sobre la enseñanza, sin dejar de encontrar distintas dificultades propias de este tipo de ambientes virtuales. Estos desafíos fueron asumidos día a día, mediados por tensiones y dificultades de conectividad, encuentros en línea y acercamientos a la realidad muy marcada por la crisis de sanitaria, que exigió garantizar la comunicación y seguir con el cumplimiento de los objetivos de cada espacio académico.

A partir de ello, este escrito capitular devela cómo los docentes y estudiantes, haciendo uso de su capacidad de resiliencia, empezaron a acomodarse para responder a sus responsabilidades y exigencias empleado diversos canales sincrónicos, asincrónicos y plataformas como Collaborate, Teams y Cisco Webex, con cortos tiempos para aprender a manejarlas. Específicamente, se presentan distintas aproximaciones a partir de un instrumento que indaga sobre las perspectivas en relación con las transformaciones y características de los docentes universitarios del Departamento de Ciencias Básicas de la Universidad de La Salle.

Al finalizar, se concluye con una reflexión orientada a aportar, en teoría y práctica, sobre lo que se piensa y se hace, para crear opciones en el proceso de formación integral, el cual exige del profesor lasallista el acompañamiento, ser flexible, creativo y abierto para lograr en el estudiante la sensibilidad y sentido de pertenencia, además de un conjunto de habilidades y técnicas diferentes a las que usa para la enseñanza presencial, pues la tecnología está transformando directa o indirectamente el modo de enseñar, e incidiendo en las apuestas del desarrollo del pensamiento crítico, autocrítico, autorresponsable y reflexivo, para enriquecer las prácticas de enseñanza desde aspectos formativos.

Finalmente, ante este reto aún surgen preguntas que esperan su respuesta, y entre ellas tenemos: ¿qué otras herramientas y elementos tecnológicos debemos conocer como docentes universitarios que buscan la calidad en cada espacio que acompañamos?, ¿con qué tiempos contamos para lograr aprendizajes reales y no copia o trabajos desarrollados por otros?, ¿qué aspectos estamos dejando pasar por alto en este nuevo proceso de interacción virtual?, ¿los estudiantes cuentan con la actitud y aptitud para lograr su aprendizaje en espacios mediados por la tecnología?, ¿cuáles son las aplicaciones que usa el estudiante para estar al día con las exigencias de cada espacio académico?, y ¿es posible ofrecer una educación de alta calidad con las condiciones de conectividad desde móviles o tecnologías poco avanzadas con las que cuentan nuestros estudiantes? 


\section{Agradecimientos}

A los coordinadores de las áreas de Matemáticas, Física y Química por el aporte de la información que permitió realizar estos análisis, así como a los diferentes docentes que aportaron sus experiencias para permitir que este ejercicio sea descrito en este capítulo y que aporte al crecimiento y adaptación de las comunidades educativas asociadas a los procesos de enseñanza-aprendizaje de las ciencias exactas y naturales.

\section{Referencias}

Molinero, M. C., \& Chávez, U. (2019). Herramientas tecnológicas en el proceso de enseñanza-aprendizaje en estudiantes de educación superior. RIDE Revista Iberoamericana para la Investigación y el Desarrollo Educativo, 10(19), e005. https://doi.org/10.23913/ride.v10i19.494

Polya, G. (1965). Cómo plantear y Resolver problemas. Editorial Trillas.

Pozo, J. (1993). Teorías cognitivas del aprendizaje. Morata.

Universidad de La Salle. (2007). Acuerdo No. 007 de marzo 21 de 2007. Proyecto Educativo Institucional-PEUL 2007. Expedido por el Consejo Superior. Universidad de La Salle. Bogotá, Colombia. https:// ciencia.lasalle.edu.co/cgi/viewcontent.cgi?article=1000\&context=peul

Universidad de La Salle. (2019). Proyecto Educativo Departamento de Ciencias Básicas PED. Vicerrectoría Académica. Departamento de Ciencias Básicas. Universidad de La Salle. Bogotá, Colombia. https:// ciencia.lasalle.edu.co/planeamiento_estrategico/19/ 